\title{
DESIGN AND FABRICATION OF EPICYCLIC INTERNAL GEAR PUMP
}

\author{
Omkar S. Pawar ${ }^{1}$, Sushil C. Suryavanshi ${ }^{2}$, Suyog B. Khamkar ${ }^{3}$, Paresh D. Shinde ${ }^{4}$, Prof. A.S. Raut ${ }^{5}$ \\ *1,2,3\&4 Student in Mechanical Engineering Department of Rajendra Mane college of Engineering and Technology, \\ Ambav (Ratnagiri) Mumbai University, India \\ ${ }^{5}$ Assistant Professor in Mechanical Engineering Department of Rajendra Mane college of Engineering and Technology, \\ Ambav (Ratnagiri) Mumbai University, India, \\ ${ }^{*}$ Email: omkar.pawar657@gmail.com, ${ }^{2}$ Email: sushilsuryavanshi7@gmail.com
}

*Corresponding Author: -

Email: omkar.pawar657@gmail.com

\begin{abstract}
: -
This paper gives outlines Epicyclic internal gear pump where one sun gear is meshed with three planet gears to achieve variable discharge rate as per requirement. This paper describes techniques for the design, construction, and testing of a Epicyclic internal gear pump. In many applications it is required to drive the actuators hydraulic cylinder or hydraulic motors at variable speed. This is only possible by variable discharge from a variable displacement pump (this pump has very high cost approx Rs.90000/-) so it is not possible to use it. One method employed is to use a pump of higher discharge capacity. But higher capacity means higher cost and higher power consumption. Hence there is need of special pump system at low cost so that the requirement of variable discharge is met easily without much cost and set up.
\end{abstract}

Keywords: "Epicyclic gear, Crescent pump, Variable discharge." 


\section{INTRODUCTION}

This system comprises of three internal gear crescent pumps mounted in parallel around epi-cyclic gear train i.e. the sun gear of the drive train drives the planet gears mounted on the input shaft of each gear pump. The input to all three gear pumps come from a common tank whereas the output from the gear pumps is delivered to a common manifold thus it is possible to get maximum discharge when needed.

The minimum output available is that of one pump. Maximum output available is that of three pumps. This is possible as each of pumps is capable of being de-coupled from circuit.

\section{PRINCIPLE}

The system entitled - EPICYCLIC INTERNAL GEAR PUMP works on principle of epicyclic gear train. The basic idea is to get maximum discharge as well as minimum discharge as per requirement. It is possible as each of pumps is capable of being de-coupled from circuit.

\section{WORKING PROCEDURE}

Basic working of Internal Gear Pump:

It is rotary flow positive displacement pump. It is more advantageous due to low speed and inlet pressure requirement. It consist of one external gear and one internal gear that meshes with each other and with (as shown in fig. b)or without crescent shaped partition (as shown in fig. a.).

When the gears disengage on the inlet side liquid comes into the pump and forces out discharge port by the meshing of the gears.

\section{Applications:}

Petrochemical, marine, terminal unloading, asphalt, chemical and general industries applications for transfer, lubrication, processing and low-pressure hydraulic fluids.

\section{Advantages:}

Compact size, low speed and inlet pressure requirement.

\section{Disadvantages:}

It can be damage while pumping large suspended solids.

It can handle small suspended particles but it causes gradual wear $\&$ loose performance.

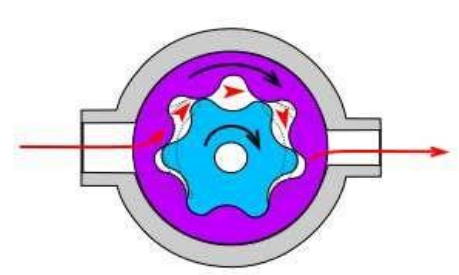

Fig. a

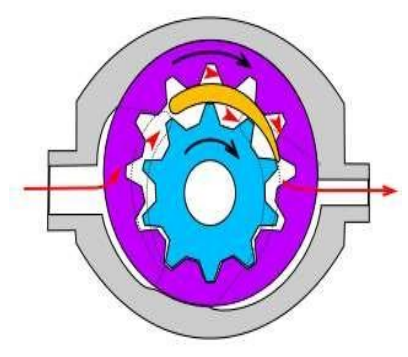

Fig. b

\section{PROJECT SETUP}

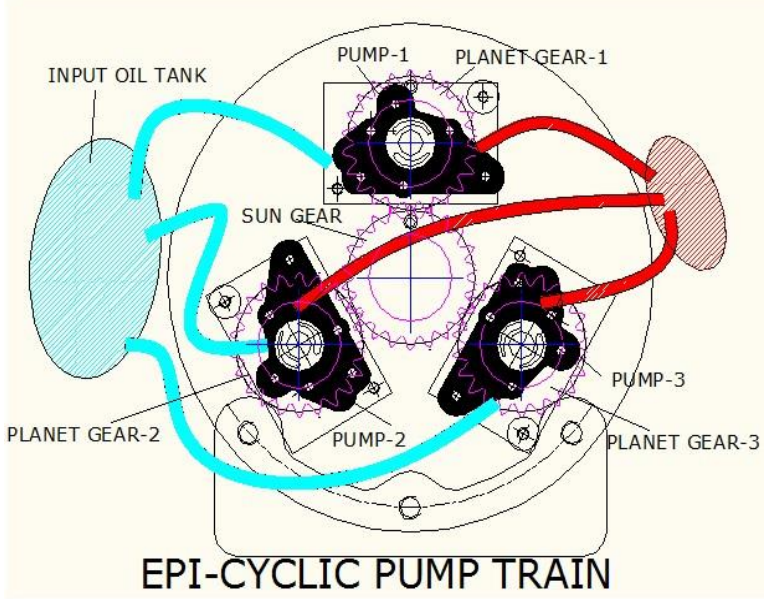

Fig. 1 Detail information of each component of the pump. 


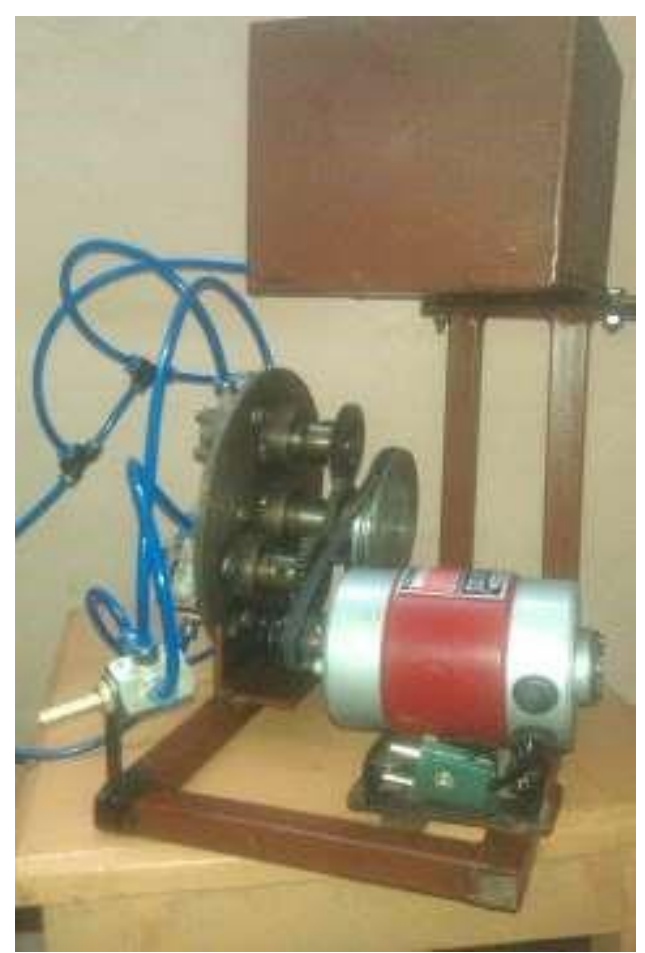

Fig. Project Setup (Side view)

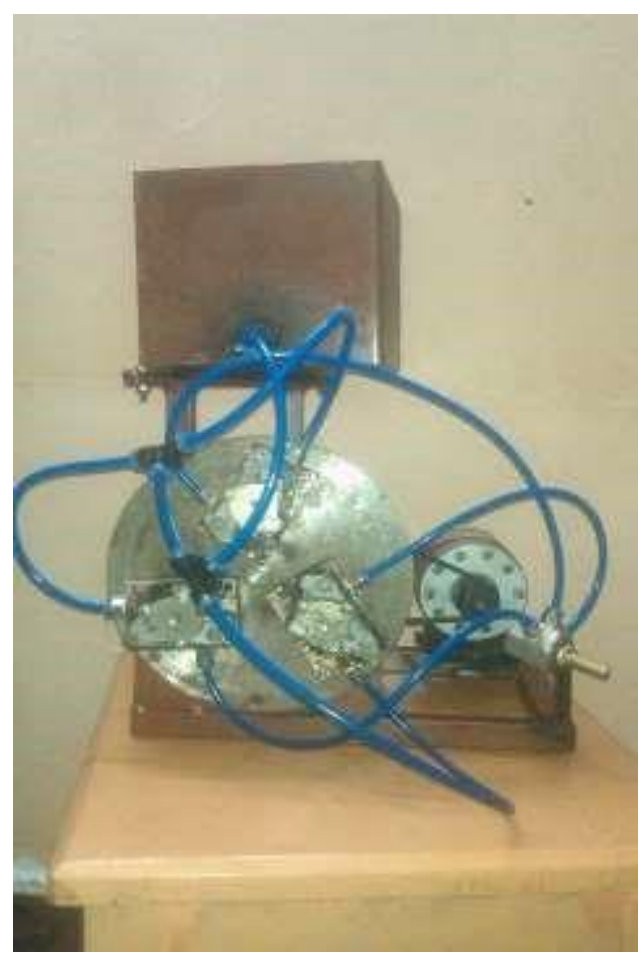

Fig. Project Setup (Front view)

\section{GENERAL ASSUMPTION IN DESIGN OF MACHINE}

\section{MOTOR SELECTION}

Thus, selecting a motor of the following specifications

Single phase AC motor

Commutator motor

TEFC construction

Power $=1 / 15 \mathrm{hp}=50$ watt

Speed $=0-6000 \mathrm{rpm}($ variable $)$

\section{DESIGN OF BELT DRIVE}

Selection an open belt drive using V-belt;

Reduction ratio $=5$

Planning a 1 stage reduction;

A) Motor pulley $(\phi \mathrm{D} 1)=20 \mathrm{~mm}$

B) Main shaft pulley $(\phi$ D2 $)=100 \mathrm{~mm}$

INPUT DATA

Input Power $=0.05 \mathrm{KW}$

Input Speed $=1000 \mathrm{rpm}$

Centre Distance $=210 \mathrm{~mm}$

Max Belt Speed $=1600 \mathrm{~m} / \mathrm{min}=26.67 \mathrm{~m} / \mathrm{s}$

Groove Angle $(2 \beta)=400$

Coefficient of Friction between belt and pulley $=0.25$

Allowable Tensile Stress $=8 \mathrm{~N} / \mathrm{mm} 2$

Section of belt section

Ref Manufacturers Catalogue

\begin{tabular}{|l|l|l|l|l|}
\hline C/S SYMBOL & $\begin{array}{l}\text { USUǍ LOAL } \\
\text { OF DRIVE } \\
(\mathrm{KW})\end{array}$ & $\begin{array}{l}\text { NOMINAL TOP } \\
\text { WIDTH }(\mathrm{W} \mathrm{mm})\end{array}$ & $\begin{array}{l}\text { NOMINAL } \\
\text { THICKNESS T } \\
\text { mm }\end{array}$ & WEIGHT DER METER Kgf \\
\hline FZ & $0.03-0.15$ & 6 & 4 & 0.05 \\
\hline
\end{tabular}

$\operatorname{Sin} \propto \quad=\frac{\mathrm{O}_{2} \mathrm{M}}{\mathrm{O}_{1} \mathrm{O}_{2}}=\frac{\mathrm{R}_{2}-\mathrm{R}_{1}}{\mathrm{x}}=\frac{\mathrm{D}_{2}-\mathrm{D}_{1}}{2 \mathrm{x}}=\frac{100-20}{2 * 210}$

$\Rightarrow \propto \quad=10.980$

Angle of lap on smaller pulley i.e. motor pulley;

$$
\begin{aligned}
\theta o & =180-2 \propto \\
& =180-2(10.98) \\
\theta & =158.04 \\
\Rightarrow \theta & =2.75^{\circ}
\end{aligned}
$$


Now;

Mass of belt $/$ meter length $=0.05 \mathrm{kgf}$

$\Rightarrow$ Centrifugal Tension $\left(\mathrm{T}_{\mathrm{c}}\right)=\mathrm{Mv} 2$

$\Rightarrow \mathrm{T}_{\mathrm{c}}=0.05$ (26.67) 2

$\Rightarrow \mathrm{T}_{\mathrm{c}}=35.56 \mathrm{~N}$

Max Tension in belt $(T)=f_{\text {all }} *$ Area

$$
=8 * 20
$$

$=160 \mathrm{~N} / \mathrm{mm} 2$

A) Tension in Tight side of belt $=\mathrm{T} 1=\mathrm{T}-\mathrm{T}_{\mathrm{c}}$

$$
\begin{array}{ll}
=160-35.56 & \\
& \mathrm{~T} 1=124.4 \mathrm{~N}
\end{array}
$$

B) Tension in slack side of belt $=\mathrm{T} 2$

$$
\begin{aligned}
& 2.3 \log \frac{\mathrm{T}_{1}}{\mathrm{~T}_{2}}=\theta * \mu * \operatorname{cosec} \beta \\
& =0.25 * 2.8 * \operatorname{cosec} \\
& \log \frac{\mathrm{T}_{1}}{\mathrm{~T}_{2}}=0.86 \\
& \Rightarrow \frac{\mathrm{T}_{1}}{\mathrm{~T}_{2}}=7.75 \\
& \Rightarrow \mathrm{T} 2=16 \mathrm{~N}
\end{aligned}
$$

POWER TRANSMITTING CAPACITY OF BELT;

$\mathrm{P}=(\mathrm{T} 1-\mathrm{T} 2) \mathrm{V}$

$=(124.24-16) 26.67$

$\mathrm{P}=3.13 \mathrm{KW}$

$\Rightarrow$ Belt can safely transmit $0.05 \mathrm{kw}$ power SELECTION OF BELT.

Selection of belt 'FZ $6 * 600$ ' from std manufacturers catalogue

MAKE: HELICORD

\section{RESULT TABLE}

\begin{tabular}{|l|l|l|}
\hline 1. & BELT SELECTED & FZ $6 * 600$ \\
\hline 2. & Tight side Tension & T1 $=124.24 \mathrm{~N}$ \\
\hline 3. & Slack side Tension & T2 $=16 \mathrm{~N}$ \\
\hline 4. & Motor pulley did. $(\phi$ D1) & D1 $=20 \mathrm{MM}$ \\
\hline 5. & Pulley $($ a) diameter $(\phi D 2)$ & D2 $=100 \mathrm{MM}$ \\
\hline
\end{tabular}

CHECK FOR TORSIONAL SHEAR FAILURE OF SHAFT.

Assuming minimum section diameter on input shaft $=16 \mathrm{~mm}$

$$
\begin{aligned}
& \Rightarrow \mathrm{d}=16 \mathrm{~mm} \\
& \mathrm{~T}_{\mathrm{d}}=\Pi / 16^{*}\left(\mathrm{f}_{\mathrm{s}}\right)_{\text {act }} * \mathrm{~d} 3 \\
& \Rightarrow\left(\mathrm{f}_{\mathrm{s}}\right)_{\text {act }}=\frac{16 * \mathrm{~T}_{\mathrm{d}}}{\pi * \mathrm{~d}^{3}} \\
& \quad=\frac{16 * 4.84 * 10^{3}}{\pi * 16^{3}} \\
& \Rightarrow\left(\mathrm{f}_{\mathrm{s}}\right)_{\text {act }}=6.01 \mathrm{~N} / \mathrm{mm} 2
\end{aligned}
$$

As $\quad\left(f_{\mathrm{s}}\right)_{\text {act }}<\left(\mathrm{f}_{\mathrm{s}}\right)_{\text {all }}$

$\Rightarrow \mathrm{I} / \mathrm{P}$ shaft is safe under torsional load

\section{DESIGN OF KEY}

Selecting parallel key from standard data book for given application.

\begin{tabular}{|l|l|l|}
\hline For Shaft Diameter & Above Upto & 17 \\
& & 22 \\
\hline Key cross section & Width Height & 6 \\
& & 6 \\
\hline
\end{tabular}

Material of key 'EN9' $S_{\text {ult }}=520 \mathrm{~N} / \mathrm{mm} 2$

$\mathrm{S}_{\text {yield }}=340 \mathrm{~N} / \mathrm{mm} 2$

$\Rightarrow\left(\mathrm{f}_{\mathrm{s}}\right)_{\text {all }}=85 \mathrm{~N} / \mathrm{mm} 2$

$\Rightarrow(\mathrm{fs})_{\text {all }}=170 \mathrm{~N} / \mathrm{mm} 2$

$\Rightarrow$ Selecting parallel key;

6* 6*30 
Check for direct shear failure of key: -

$$
\begin{aligned}
& \quad \mathrm{T}=\mathrm{L} * \mathrm{~d} / 2 * \mathrm{t} / 2 *\left(\mathrm{f}_{\mathrm{s}}\right)_{\text {act }} \\
& \Rightarrow 4.84 \times 103=\frac{30 \times 6 \times 20 \times\left(\mathrm{f}_{\mathrm{s}}\right)_{\text {act }}}{2.2} \\
& \Rightarrow\left(\mathrm{f}_{\mathrm{s}}\right)_{\text {act }}=\frac{4.84 \times 103 \times 2}{30 * 6 * 20} \\
& \Rightarrow\left(\mathrm{f}_{\mathrm{s}}\right)_{\text {act }}=2.68 \mathrm{~N} . \mathrm{mm} 2 \\
& \text { As }\left(\mathrm{f}_{\mathrm{s}}\right)_{\text {act }}<\left(\mathrm{f}_{\mathrm{s}}\right)_{\text {all }} .
\end{aligned}
$$

$\Rightarrow$ Key is safe under shear load

Check for crushing failure of key

$$
\begin{aligned}
& \mathrm{T}=\mathrm{L} * \mathrm{~d} / 2 * \mathrm{t} / 2 *\left(\mathrm{f}_{\mathrm{s}}\right)_{\text {act }} \\
& 7.66 \times 103=30 * 20 / 2 * 6 / 2 *\left(\mathrm{f}_{\mathrm{s}}\right)_{\text {act }} \\
& \Rightarrow\left(\mathrm{f}_{\mathrm{s}}\right)_{\text {act }}=\frac{4.84 \times 103 \times 2 \times 2}{30 * 20 * 6} \\
& \Rightarrow\left(\mathrm{f}_{\mathrm{s}}\right)_{\text {act }}=5.367 \mathrm{~N} . \mathrm{mm} 2 \\
& \text { As }\left(\mathrm{f}_{\mathrm{s}}\right)_{\text {act }}<\left(\mathrm{f}_{\mathrm{s}}\right)_{\text {all }}
\end{aligned}
$$

$\Rightarrow$ Key is safe under crushing load.

\section{DESIGN OF PLANETARY SPUR GEAR BOX}

The multi spindle drilling attachment has an drive train in the form of planetary gear system comprising of the central sun gear and three planet gears which drive the three individual spindles on which drill chucks are mounted. The following dimensions are assumed for the gear drive train,

Sun gear

Module $=1.5 \mathrm{~mm}$

No. of teeth $=32$

Planet gear

Module $=1.5 \mathrm{~mm}$

No. of teeth $=32$

Power $=0.5 \mathrm{HP}=375$-watt Speed $=480 \mathrm{rpm} \mathrm{b}=10 \mathrm{~mm} \mathrm{~T}_{\text {design }}=4.84 \mathrm{~N} . \mathrm{m}$

$\left(\mathrm{S}_{\mathrm{ult}}\right)$ pinion $=\left(\mathrm{S}_{\mathrm{ult}}\right)$ gear $=600 \mathrm{~N} / \mathrm{mm} 2$

Service factor $\left(\mathrm{C}_{\mathrm{s}}\right)=1.5$

$$
\begin{aligned}
& \text { Now; } T=\left(P_{t}\right) * \frac{d_{p}}{2} \\
& \Rightarrow\left(P_{t}\right)=201.6 \mathrm{~N} . \\
& \left(P_{\text {eff }}\right)=\frac{P_{t} * C_{s}}{1.5}=\frac{750 * 1.5}{1.5} \\
& \text { Now; } \quad C_{v}=\frac{3}{3+v} \\
& v=\Pi D N=\frac{\Pi \times 48 \times 10-3 \times 480}{60}=1.2 \mathrm{~m} / \mathrm{sec} \\
& \Rightarrow C_{v}=1.2 \\
& \Rightarrow P_{\text {eff }}=\frac{201.6 * 1.5}{1.2} * 1.5 \\
& P_{\text {eff }}=378
\end{aligned}
$$

Lewis Strength equation

$$
\begin{aligned}
& \text { WT }=\mathrm{S} * \mathrm{~b} * \mathrm{y} * \mathrm{~m} \\
& \text { Where; } \\
& \mathrm{y}=\frac{0.484-2.86}{\mathrm{z}} \\
& \Rightarrow \mathrm{y}_{\mathrm{p}}=\frac{0.484-2 . .86}{32}=0.394 \\
& \Rightarrow \mathrm{s}_{\mathrm{vb}}=0.394
\end{aligned}
$$

Pinion and gear both are of same material and with same number of teeth hence

$$
\begin{aligned}
& \mathrm{s}_{\mathrm{yp}}=\mathrm{s}_{\mathrm{yg}}=236.4 \\
& \mathrm{WT}=\left(\mathrm{s}_{\mathrm{yp}}\right) * \mathrm{~b} * \mathrm{~m} \\
& =236.4 * 10 \mathrm{~m} * \mathrm{~m} \\
& \mathrm{WT}=2364 \mathrm{~m} 2 \\
& \text { Equation }(\mathrm{A}) \&(\mathrm{~B}) \\
& 2364 \mathrm{~m} 2=378 \\
& \Rightarrow \mathrm{m}=0.39
\end{aligned}
$$


Selecting standard module $=1.5 \mathrm{~mm}$

GEAR DATA

No. of teeth $=32$

Module $=1.5 \mathrm{~mm}$

Addendum diameter $=51 \mathrm{~mm}$

Deddendum diameter $=44.25 \mathrm{~mm}$

DESIGN OF PLANET SPINDLES

MATERIAL SELECTION: - Ref: - PSG (1.10 \& 1.12) + (1.17)

\begin{tabular}{|l|l|c|}
\hline DESIGNATION & $\begin{array}{l}\text { ULTIMATE TENSILE } \\
\text { STRENGTH N/mm2 }\end{array}$ & $\begin{array}{c}\text { YEILD STRENGTH } \\
\text { N/mm2 }\end{array}$ \\
\hline EN 24 & 800 & 680 \\
\hline
\end{tabular}

\section{ASME CODE FOR DESIGN OF SHAFT.}

Since the loads on most shafts in connected machinery are not constant, it is necessary to make proper allowance for the harmful effects of load fluctuations According to ASME code permissible values of shear stress may be calculated from various relations.

Considering minimum of the above values;

$$
\begin{gathered}
=0.18 \times 800 \\
=144 \mathrm{~N} / \mathrm{mm} 2 \mathrm{OR} \\
\left(f_{\mathrm{s}}\right) \mathrm{max}=0.3 \mathrm{f}_{\mathrm{yt}} \\
=0.3 \times 680=204 \mathrm{~N} / \mathrm{mm}
\end{gathered}
$$

$$
\Rightarrow\left(\mathrm{f}_{\mathrm{s}}\right) \max =144 \mathrm{~N} / \mathrm{mm} 2
$$

DESIGN OF INPUT SHAFT.

MATERIAL SELECTION: - Ref.:- PSG (1.10 \& 1.12) + (1.17)

\begin{tabular}{|l|l|l|}
\hline & $\begin{array}{l}\text { ULTIMATE TENSILE } \\
\text { STRENGTH } \\
\text { N/mm2 }\end{array}$ & YEILD STRENGTH N/mm2 \\
\hline EN 24 & 800 & 680 \\
\hline
\end{tabular}

ASME CODE FOR DESIGN OF SHAFT.

Since the loads on most shafts in connected machinery are not constant, it is necessary to make proper allowance for the harmful effects of load fluctuations

According to ASME code permissible values of shear stress may be calculated from various relations.

$$
\begin{gathered}
=0.18 \times 800 \\
=144 \mathrm{~N} / \mathrm{mm} 2 \mathrm{OR} \\
\left(\mathrm{f}_{\mathrm{s}}\right)_{\max }=0.3 \mathrm{f}_{\mathrm{yt}} \\
=0.3 \times 680=204 \mathrm{~N} / \mathrm{mm} 2
\end{gathered}
$$

Considering minimum of the above values;

$$
\Rightarrow\left(\mathrm{f}_{\mathrm{s}}\right)_{\max }=144 \mathrm{~N} / \mathrm{mm} 2
$$

Shaft is provided with key way; this will reduce its strength. Hence reducing above value of allowable stress by $25 \%$

$$
\Rightarrow\left(\mathrm{f}_{\mathrm{s}}\right)_{\max }=108 \mathrm{~N} / \mathrm{mm} 2
$$

This is the allowable value of shear stress that can be induced in the shaft material for safe operation.

\section{TO CALCULATE INTERMEDIATE SHAFT TORQUE}

POWER $=\frac{2 \Pi N T}{60}$

Motor is 50-watt power, run at $5000 \mathrm{rpm}$, connected to intermediate shaft by belt pulley arrangement with reduction ratio $1: 5$

Hence input to input shaft $=1000 \mathrm{rpm}$

$$
\begin{aligned}
& \Rightarrow \mathrm{T}=\frac{600 \mathrm{P}}{2 \pi \mathrm{N}}=\frac{60 * 50}{2 * \pi * 1000} \\
& \Rightarrow \mathrm{T}=0.48 \mathrm{~N}-\mathrm{m} \\
& \Rightarrow \mathrm{T}_{\text {design }}=0.48 \mathrm{~N}-\mathrm{m}
\end{aligned}
$$




\section{CHECK FOR TORSIONAL SHEAR FAILURE OF SHAFT.}

But as per manufacturing considerations we have an $\mathrm{H} 6 \mathrm{~h} 7$ fit between the pulley and shaft and to achieve this tolerance boring operation is to be done and minimum boring possible on the machine available is $16 \mathrm{~mm}$ hence consider the minimum section on the shaft to be $16 \mathrm{~mm}$

Assuming minimum section diameter on input shaft $=16 \mathrm{~mm}$

$\Rightarrow \mathrm{I} / \mathrm{P}$ shaft is safe under torsional load.

$$
\begin{aligned}
& \mathrm{T}_{\mathrm{d}}=\Pi / 16 *\left(\mathrm{f}_{\mathrm{s}}\right)_{\text {act }} * d^{3} \mathrm{~d}=16 \mathrm{~mm} \\
& \Rightarrow\left(f_{s}\right)_{\text {act }} \quad=\frac{16 * T_{d}}{\Pi * d^{3}} \\
& \Rightarrow\left(\mathrm{f}_{\mathrm{s}}\right)_{\text {act }}=0.6 \mathrm{~N} / \mathrm{mm} 2 \\
& =\left(16 * 0.48 *(10)^{\wedge} 3\right) /\left(\Pi \times(16)^{\wedge} 3\right) \\
& \text { As }\left(\mathrm{f}_{\mathrm{s}}\right)_{\text {act }}<\left(\mathrm{f}_{\mathrm{s}}\right)_{\text {all }}
\end{aligned}
$$

\section{DESIGN (SELECTION OF INPUT SHAFT BALL BRG}

In selection of ball bearing the main governing factor is the system design of the drive i.e.; the size of the ball bearing is of major importance; hence we shall first select an appropriate ball bearing first select an appropriate ball bearing first taking into consideration convenience of mounting the planetary pins and then we shall check for the actual life of ball bearing.

BALL BEARING SELECTION.

Series 60

\begin{tabular}{|l|l|l|l|l|l|l|l|l|}
\hline ISI NO & $\begin{array}{l}\text { Brg. (SKF) } \\
\text { Series No. }\end{array}$ & d & D1 & D & D2 & B & \multicolumn{2}{|l|}{ Basic capacity } \\
\hline & & & & & & & C kgf & Co Kgf \\
\hline 20A C04 & 6004 & 20 & 23 & 42 & 36 & 12 & 4650 & 2850 \\
\hline
\end{tabular}

$\mathrm{P}=\mathrm{XF} \mathrm{F}_{\mathrm{r}}+\mathrm{YF}_{\mathrm{a}}$.

Where;

$\mathrm{P}=$ Equivalent dynamic load, $(\mathrm{N})$

$\mathrm{X}=$ Radial load constant

$\mathrm{F}_{\mathrm{r}}=$ Radial load $(\mathrm{H})$

$\mathrm{Y}=$ Axial load contact

$\mathrm{F}_{\mathrm{a}}=$ Axial load $(\mathrm{N})$

In our case;

Radial load $\mathrm{Fr}=\mathrm{T}_{1}+\mathrm{T}_{2}=124.4+16=140.4 \mathrm{~N}$

$\mathrm{Fa}=0$

$\mathrm{P}=1 * 140.4 \mathrm{~N}$

$\Rightarrow \mathrm{L}=(\mathrm{C} / \mathrm{p}) \mathrm{p}$

Considering 4000 working hours

$$
\begin{aligned}
& \mathrm{L}=\frac{60 \mathrm{nLh}}{106} \quad=240 \mathrm{~m} \text { rev } \\
& \Rightarrow 240=[\mathrm{C} / 140.4]^{3} \\
& \Rightarrow \mathrm{C}=872.5 \mathrm{~N}
\end{aligned}
$$

As required dynamic of bearing is less than the rated dynamic capacity of bearing;

$\Rightarrow$ Bearing is safe.

\section{DESIGN OF GEAR DRUM HUB: -}

Brake drum hub can be considered to be a hollow shaft subjected to torsional load.

Material selection:

\begin{tabular}{|l|l|l|}
\hline Designation & Ultimate Tensile strength N/mm2 & Yield strength N/mm2 \\
\hline CI & 500 & 380 \\
\hline
\end{tabular}

As Per ASME Code; (FACTOR OF SAFETY=3)

$\Rightarrow\left(\mathrm{f}_{\mathrm{s}}\right)_{\max }=166.6 \mathrm{~N} / \mathrm{mm} 2$

Check for torsional shear failure: -

$$
\begin{aligned}
& \mathrm{T}=\frac{\pi *\left(\mathrm{f}_{\mathrm{s}}\right)_{\text {act }}}{16} *\left[\frac{\mathrm{Do}^{4}-\mathrm{Di}^{4}}{\mathrm{Do}}\right] \\
& 0.48 \times 103=\frac{\pi *\left(\mathrm{f}_{\mathrm{s}}\right)_{\text {act }}}{16} *\left[\frac{56^{4}-35^{4}}{56}\right] \\
& \Rightarrow\left(\mathrm{f}_{\mathrm{s}}\right)_{\text {act }}=0.162 \mathrm{~N} / \mathrm{mm} 2 \\
& \text { As; }\left(\mathrm{f}_{\mathrm{s}}\right)_{\text {act }}<\left(\mathrm{f}_{\mathrm{s}}\right)_{\text {all }}
\end{aligned}
$$


$\Rightarrow$ Hub is safe under torsional load

DESIGN OF SPUR GEAR PAIR FOR DRIVE FROM INPUT SHAFT TO PLANET SHAFT

Power $=01 / 15 \mathrm{HP}=50$ watts

Speed $=1000 \mathrm{rpm}$

$\mathrm{b}=10 \mathrm{~m}$

$\mathrm{T}_{\text {design }}=0.48 \mathrm{~N} . \mathrm{m}$

$\left(\mathrm{S}_{\mathrm{ult}}\right)$ pinion $=\left(\mathrm{S}_{\mathrm{ult}}\right)$ gear $=400 \mathrm{~N} / \mathrm{mm} 2$

Service factor $(\mathrm{Cs})=1.5 \mathrm{~d}_{\mathrm{p}}=55.5$

Considering 1.5 module gear with 37 teeth

$\mathrm{T}=\mathrm{T}_{\text {design }}=0.48 \mathrm{~N}-\mathrm{m}$

Now; $\mathrm{T}=\mathrm{P}_{\mathrm{t}} * \mathrm{~d}-{ }_{2}^{\mathrm{p}}$

$\Rightarrow \mathrm{P}_{\mathrm{t}}=17.3 \mathrm{~N}$.

Neglecting effect of $\mathrm{C}_{\mathrm{v}}$ as speed is very low

$$
\mathrm{P}_{\mathrm{eff}}=\frac{\mathrm{P}_{\mathrm{t}} \times \mathrm{C}_{\mathrm{s}}}{\mathrm{C}_{\mathrm{v}}}=\frac{17.3 * 1.5}{\mathrm{C}_{\mathrm{v}}}
$$

$$
\mathrm{P}_{\text {eff }}=26 \mathrm{~N}
$$

Lewis Strength equation

$\mathrm{WT}=\mathrm{S} * \mathrm{~b} * \mathrm{y} * \mathrm{~m}$

Where;

$$
\begin{aligned}
& y=\frac{0.484-2.86}{z} \\
& \Rightarrow y_{p}=\frac{0.484-2 . .86}{37}=0.4060 \\
& \Rightarrow S_{y p}=162.68
\end{aligned}
$$

Pinion and gear both are of same material and with same number of teeth hence

$$
\begin{array}{r}
\mathrm{S}_{\mathrm{yp}}=\mathrm{S}_{\mathrm{yg}}=162.68 \\
\mathrm{WT}=\left(\mathrm{S}_{\mathrm{yp}}\right) * \mathrm{~b} * \mathrm{~m} \\
=162.68 * 10 \mathrm{~m} * \mathrm{~m} \\
\mathrm{WT}=1626.8 \mathrm{~m}^{2}
\end{array}
$$

Equation (A) \& (B)

Selecting standard module $=1.5 \mathrm{~mm}$

$$
1626.8 m^{2}=26
$$$$
\Rightarrow \mathrm{m}=0.1
$$

\section{SELECTED MATERIALS}

TABLE I Material Specification

\begin{tabular}{|c|c|c|c|c|c|}
\hline SR NO. & PART CODE & DESCRIPTION & QTY & SPECIFICATION & MATERIAL \\
\hline 1 & EGP-1 & BASE PLATE & 01 & & EN9 \\
\hline 2 & EGP-2 & MOTOR & 01 & 50watt,0to6000rpm & EN9 \\
\hline 3 & EGP-3 & PULLEY & 04 & & STD \\
\hline 4 & EGP-4 & MAIN SHAFT & 01 & DIA. $=16 \mathrm{~mm}$ & EN24 \\
\hline 5 & EGP-5 & MAIN SHAFT HSG & 01 & & EN9 \\
\hline 6 & EGP-6 & PLANET GEARS & 03 & MODULE $=1.5$ No. of & EN24 \\
\hline 7 & EGP-7 & SUN GEAR & 01 & Module=1.5 No. of teeth=32 & EN24 \\
\hline 8 & EGP-8 & PUMPSHAFT & 03 & & EN24 \\
\hline 9 & EGP-9 & PUMP SHAFT HSG & 03 & & EN9 \\
\hline 10 & EGP-10 & PUMP BLOCKS & 03 & & AL \\
\hline 11 & EGP-11 & PUMP & 03 & Crescent pumps & MS \\
\hline 12 & EGP-12 & BELT & 01 & & FZ $*$ 600 \\
\hline 13 & EGP-13 & INLET MANIFOLD & 01 & & AL \\
\hline 14 & EGP-14 & OUTLET MANIFOLD & 01 & & STD \\
\hline 15 & EGP-15 & MOUNTING PLATE & 01 & & STD \\
\hline 16 & EGP-16 & BOLTS & 06 & & \\
\hline
\end{tabular}




\section{VII.TESTING PROCEDURE}

1. Maintain input speed at input $=100 \mathrm{rpm}$.

2. Collect $100 \mathrm{ml}$ of oil in measuring beaker.

3. Note time for collecting $100 \mathrm{ml}$ of oil.

4. Change input speed to $200 \mathrm{rpm}$.

5. Repeat step $4 \& 5$.

6. Repeat procedure for $300 \mathrm{rpm}, 400 \mathrm{rpm}$ and $500 \mathrm{rpm}$.

\section{RESULTS}

\begin{tabular}{|l|l|l|l|l|}
\hline $\begin{array}{l}\text { SR. } \\
\text { NO. }\end{array}$ & $\begin{array}{l}\text { SPEED } \\
\text { RPM })\end{array}$ & $\begin{array}{l}\text { VOLUME IN } \\
\text { BEAKER } \\
(\mathrm{ml})\end{array}$ & $\begin{array}{l}\text { TIME } \\
\text { (SECONDS })\end{array}$ & $\begin{array}{l}\text { FLOW } \\
\text { RATE } \\
(\text { LPM })\end{array}$ \\
\hline 01 & 100 & 100 & 189 & 0.0317 \\
\hline 02 & 200 & 100 & 94 & 0.063 \\
\hline 03 & 300 & 100 & 65 & 0.092 \\
\hline 04 & 400 & 100 & 49 & 0.122 \\
\hline 05 & 500 & 100 & 40 & 0.15 \\
\hline
\end{tabular}

\begin{tabular}{|l|l|l|l|l|}
\hline $\begin{array}{l}\text { SR. } \\
\text { NO. }\end{array}$ & $\begin{array}{l}\text { SPEED } \\
\text { RPM })\end{array}$ & $\begin{array}{l}\text { ACTUAL } \\
\text { FLOW } \\
\text { RATE (LPM) }\end{array}$ & $\begin{array}{l}\text { THEORETICAL FLOW } \\
\text { RATE }\end{array}$ & $\begin{array}{l}\text { VOLUMETRIC } \\
\text { EFFICIENCY }\end{array}$ \\
\hline 01 & 100 & 0.0317 & 0.036 & 86.35 \\
\hline 02 & 200 & 0.063 & 0.073 & 86.82 \\
\hline 03 & 300 & 0.092 & 0.11 & 83.7 \\
\hline 04 & 400 & 0.122 & 0.147 & 83.2 \\
\hline 05 & 500 & 0.15 & 0.183 & 81.65 \\
\hline
\end{tabular}

\section{GRAPHS}

\section{ACTUALFLOW RATE}

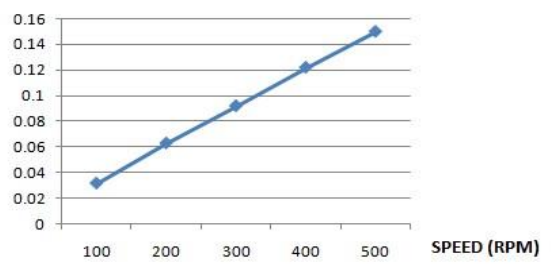

\section{VOLUMETRIC EFFICIENCY}

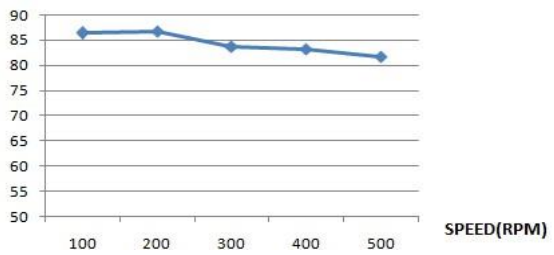
ADVANTAGES
1. Minimum discharge available by use of one pump
2. Maximum discharge is available by use of three pumps in parallel
3. Common drive from single motor using epi-cyclic gear train; so less power required.
4. Low cost of manufacturing
5. Low cost of operation
6. Low maintenance cost
7. Easily available pump units in commercial market so easy to replace parts if they fail.

\section{APPLICATIONS}

1. Spring making machines

2. Sheet metal shearing machines

3. Sheet rolling machines

4. Sheet forming and bending machines

5. Conveyor plants

\section{CONCLUSION}

The pump must be of less cost as well as compact in size so that it can be use for many applications. By considering the requirements of industries we designed a pump which will fulfill their needs. It is approximately $70 \%$ cheaper in cost as compare to other conventional pumps available in market. As the parts of pump are easily available in market; they can be replaced when they get damaged. 


\section{REFERENCES}

[1].PLANETARY GEAR PUMP, Wilson A. Burtis,5011 Harvard Ave, Westminster, Calif, Filed Oct. 24, 1963 , Ser.No. 318,670, 7 Claims. (Cl. (103-126).

[2].PISTON PUMP WITH PLANETARY GEAR DRIVE, Albert A. Zalis, Warren, Mass Appl.No.: 843, 955 , Field Oct $20,1977$.

[3].OIL PUMP FOR CONTROLLING PLANETARY SYSTEM TORQUE, Perry Edward Phelan, Harsens Island , MI (U.S.); Joseph Palazzolo, Livonia, MI(U.S.), Appl. No.: 10/040,848, Filled: Dec. 28, 2001.

[4].Machine Design by R S Khurmi .

[5].Elements of Machine Design by V.B Bhandari.

[6].http://upload.wikimedia.org/wikipedia/commons/c/c4/Gear_pump.png

[7].http://upload.wikimedia.org/wikipedia/commons/7/78/Gear_pump_2.png .

[8].P.S.G. Data Book. 